\title{
Using HIV Voluntary Counseling and Testing Data for Monitoring the Uganda HIV Epidemic, 1992-2000
}

\author{
Fulgentius Baryarama, MSc, *† Rebecca E. Bunnell, ScD, MEd, * Raymond L. Ransom, MPH, * \\ John Paul Ekwaru, MSc, * Josephine Kalule, MA, $†$ Esther B. Tumuhairwe, BStat, $\dagger$ \\ Jonathan H. Mermin, MD, MPH*
}

Objective: To assess trends in the prevalence of HIV infection among voluntary counseling and testing (VCT) clients in Uganda and to describe the utility of VCT data for monitoring the HIV epidemic in 1992-2000.

Methods: We analyzed routinely collected data from first-time VCT clients not reporting illness as a reason for testing. We developed a model adjusting for test site, couple testing, and premarital testing, assessed trends in adjusted prevalence of HIV infection and shifts in age-specific peak prevalence, and compared antenatal clinic (ANC) surveillance data and VCT prevalence trends.

Results: Among 201,741 clients, adjusted prevalence of HIV infection declined from $23 \%$ in 1992 to $13 \%$ in $2000(P<0.001)$ (men, $17 \%-9 \%[P<0.001]$; women, 31\%-18\% $[P<0.001])$. The prevalence declined for all age groups except men older than 39 years and women older than 34 years. The prevalence increased for women older than 39 years $(P<0.003)$. Between 1992 and 2000, peak prevalence declined for both men ( $31 \%$ to $24 \%$ ) and women ( $44 \%$ to $41 \%$ ), whereas the age at which the peak occurred increased for both men ( 36 to 41 years) and women ( 31 to 36 years). VCT and ANC prevalence trends were similar.

Conclusion: In Uganda, the prevalence of HIV infection among male and female VCT clients declined from 1992 to 2000, similar to ANC surveillance data, but did not decline in older age groups. In regions with well-established VCT programs, VCT data may provide a useful and convenient tool for monitoring the HIV epidemic.

Key Words: HIV, prevalence, voluntary counseling and testing (VCT), antenatal clinic (ANC), monitoring, surveillance, Africa

(J Acquir Immune Defic Syndr 2004;37:1180-1186)

$\mathrm{N}$ early 2 decades after AIDS was first identified in Africa, substantial resources for prevention and care interventions are becoming available. ${ }^{1-5}$ Increased funding combined

Received for publication May 19, 2003; accepted March 2, 2004.

From *CDC-Uganda, Global AIDS Program, National Center for HIV/AIDS, STD, and TB, Centers for Disease Control and Prevention, Atlanta, GA; and $\uparrow$ AIDS Information Center, Kampala, Uganda.

Reprints: Rebecca Bunnell, CDC-Uganda, c/o Global AIDS Program, National Center for HIV/AIDS, STD, and TB, CDC, 1600 Clifton Road NE, Atlanta, GA 30333 (e-mail: rbunnell@cdc.gov).

Copyright $@ 2004$ by Lippincott Williams \& Wilkins with effective interventions, such as nevirapine for the prevention of mother-to-child transmission (PMTCT) ${ }^{6,7}$ and voluntary counseling and testing (VCT), ${ }^{8,9}$ should result in widespread utilization of these interventions. As access to PMTCT services grows, the feasibility and ethics of collecting anonymous antenatal clinic (ANC) data for HIV surveillance are being questioned. Sources of supplementary surveillance data need to be identified and assessed so that our ability to monitor progress in confronting the HIV epidemic is not diminished, particularly in sub-Saharan Africa where the combination of high prevalence of HIV infection ${ }^{10}$ and high fertility ${ }^{11}$ calls for rapid expansion of PMTCT interventions. In countries like Uganda, with well-established VCT programs, VCT data may provide a useful and convenient tool for monitoring the HIV epidemic. Triangulation of ANC data with VCT, PMTCT, and other programmatic data may form the foundation for surveillance and monitoring of HIV trends during an era of widely expanded programmatic interventions.

As in most countries, ANC sites currently provide most of Uganda's surveillance data. ${ }^{12}$ The strengths of ANC data to provide estimates of the prevalence of HIV infection representative for the general population include the fact that ANC attendees have engaged in unprotected sex, may belong to an age range in which HIV infection is most prevalent, provide a large sample that is not prone to demographic variability over long periods, and are less likely to reflect participation bias than other potential surveillance populations. ${ }^{13}$ However, the extent to which HIV trends among ANC attendees reflect trends in the general population has been extensively debated. ${ }^{14-17}$ Central to these discussions is the suggestion that prevalence of HIV infection based on data for ANC attendees underestimates true population prevalence, lacks sufficient stratification, and does not address trends in the prevalence of HIV infection among men or nonpregnant women. ${ }^{14}$

Although routine data from sources outside sentinel surveillance sites have been used elsewhere to monitor the epidemic, ${ }^{18}$ the usefulness of the increasing volume of VCT programmatic data from sub-Saharan Africa and elsewhere has not been explored. ${ }^{9,19,20}$ In addition to its potential utility for internal monitoring of VCT programs, VCT data may have distinct advantages that complement ANC surveillance data. 
In contrast to ANC data, VCT data include findings for both men and women, offer wide age stratification, and allow for routine collection of behavioral data including sexual history and recent risk exposures.

Uganda was the first country in sub-Saharan Africa to introduce HIV VCT, establishing services in 1990. VCT has been a cornerstone of HIV prevention and care activities in Uganda and $>500,000$ VCT sessions have been conducted since 1990 . We analyzed trends in the prevalence of HIV infection over a 9-year period (1992-2000) from a large database of Ugandan VCT clients tested at 4 established sites. We also compared VCT and ANC data from the same urban towns to assess the utility of VCT data as a supplement to ANC surveillance data.

\section{METHODS}

\section{Setting}

The AIDS Information Center (AIC) is a Ugandan nongovernmental organization formed in 1990 that offers $\mathrm{VCT}^{21}$ services at 4 main branches in Uganda's major towns of Kampala (capital city), Jinja, Mbarara, and Mbale. These 4 main branches are stand-alone VCT sites (ie, not attached to or integrated into any previously established health unit) and have operated 6 days a week since they opened. In addition to VCT, AIC provides all clients with syphilis screening, sexually transmitted disease and tuberculosis education, and an opportunity for ongoing psychosocial support through posttest clubs. Cost sharing for AIC services began in 1994 and includes an exemption policy for clients unable to pay. Awareness of AIC sites was created through a wide variety of community mobilization programs involving local government meetings and leaders, AIC drama performances, client testimonies, religious institutions, radio programs, and newspapers in local languages.

\section{HIV Testing}

From 1992 to 1996, serologic testing for HIV was conducted at Uganda's National Blood Bank using a standard double HIV ELISA testing algorithm with Western blot confirmation when the ELISA results were discordant. Since 1997, AIC has offered on-site rapid HIV testing using a sequential testing algorithm: Capillus (Trinity Biotech, Bray, Eire) HIV-1/HIV-2 as a screening assay, SeroCard (Trinity Biotech, Bray, Eire) HIV as a confirmatory assay, and Multispot (Sanofi Diagnostics Pasteur, Paris, France) HIV1/HIV-2 as a "tiebreaker." A positive specimen is defined as one that is positive by both screening and confirmatory assays or positive by screening and "tiebreaker" assays. This rapid testing algorithm has been shown to have $100 \%$ sensitivity and specificity $^{22}$ compared with standard EIA testing.

\section{Data Collection}

AIC receptionists routinely collect data from all clients on date of birth, religion, education, occupation, ethnicity, and location of residence. Counselors routinely collect data on behavioral factors, including self-reported previous HIV tests, condom use at last sex, reasons for seeking HIV testing, and type of testing (individual vs. couple). Laboratory results are recorded on client cards. Data are entered into a computerized database. During analysis, age is computed from the HIV test date and the client's reported birth date. A data manager closely supervises the data collection and entry processes. A random validation of $5 \%$ of all client data showed per record error rates of $<1 \%$ (AIC Data Validation Report, November 2000). The analyses presented in this report consisted only of secondary unlinked data analysis; no contact with human subjects occurred.

\section{Data Analysis}

Changes in client characteristics were assessed by comparing 1992 and 2000 data for linear trends across all the years (Table 1). A logistic regression model was used to adjust prevalences for other covariates (eg, sex, age, calendar test year, test site [categorical], couple testing, and premarital testing [defined as client reporting "to get married" as reason for testing]). All 2-way interactions of these variables were assessed, and the final model was obtained by stepwise backward elimination. The final model retained all the core variables including sex, age, calendar test year, test site, couple testing, and premarital testing. Adjusted prevalences by sex, age, and calendar year were then obtained by taking the mean model predicted probability for HIV positivity of clients in the category. All subsequent analyses were done using prevalences adjusted based on this model unless otherwise indicated.

Trends in overall prevalence of HIV infection by calendar year, sex, and age were assessed using the CochranArmitage trend test for adjusted grouped data weighted by the number of HIV-positive and -negative clients. Adjusted agespecific prevalence data for 1992 and 2000 were plotted to present shifts in peak prevalence for men and women. Agespecific prevalence trends for women attending ANC at 3 sentinel surveillance sites (Kampala, Jinja, and Mbarara) and for female VCT clients at AIC main branches in the same cities were compared. Although the comparisons were age specific for women aged 15-19 years, 20-24 years, and 25-49 years, no further standardization on ANC prevalence was possible because complete ANC data were not available. All analyses were conducted using SAS (version 6.12; SAS Institute, Cary, NC).

\section{RESULTS}

\section{Client Characteristics}

A total of 246,715 clients aged 15 years or older had their first HIV test performed at the 4 main branches over the period January 1992 to December 2000. From these clients, we ex- 
TABLE 1. Changes in Client Characteristics Among VCT Clients, 1992-2000

\begin{tabular}{|c|c|c|c|}
\hline Characteristics & 1992 & 2000 & $\begin{array}{l}\text { All Clients } \\
1992-2000\end{array}$ \\
\hline No. of clients & 20,991 & 18,256 & 201,741 \\
\hline Females & 45 & 49 & 49 \\
\hline \multicolumn{4}{|l|}{ Age (y) } \\
\hline 15-19 & 15 & 14 & 15 \\
\hline $20-24$ & 34 & 29 & 31 \\
\hline $25-29$ & 24 & 24 & 25 \\
\hline $30-34$ & 14 & 15 & 14 \\
\hline $35-39$ & 6 & 8 & 7 \\
\hline $40-44$ & 3 & 5 & 4 \\
\hline 45 and older & 4 & 5 & 4 \\
\hline \multicolumn{4}{|l|}{ Marital status } \\
\hline Single & 60 & 58 & 59 \\
\hline Married & 30 & 31 & 29 \\
\hline Other & 10 & 12 & 12 \\
\hline \multicolumn{4}{|l|}{ Education } \\
\hline None & 3 & 6 & 5 \\
\hline Primary & 36 & 34 & 42 \\
\hline Secondary & 53 & 51 & 45 \\
\hline Postsecondary & 9 & 9 & 9 \\
\hline \multicolumn{4}{|l|}{ HIV test site } \\
\hline Kampala & 70 & 42 & 56 \\
\hline Jinja & 24 & 25 & 20 \\
\hline Mbarara & 6 & 22 & 16 \\
\hline Mbale & 0.3 & 12 & 9 \\
\hline Couple testing & 11 & 30 & 25 \\
\hline Premarital testing & 24 & 34 & 32 \\
\hline $\begin{array}{l}\text { Used condoms at last sexual } \\
\text { encounter }\end{array}$ & 9 & 19 & 14 \\
\hline Prevalence of HIV infection & 23 & 13 & 18 \\
\hline
\end{tabular}

Data are percents unless stated otherwise. $P<0.001$ for change in distribution across all years between 1992 and 2000.

cluded 44,974 who reported illness as a reason for testing to avoid selection bias due to higher prevalence of HIV infection ( $61 \%$ vs. $14 \%$, respectively) and an increase in the proportion of such clients over time (14\% to $19 \%)$. The proportions of clients excluded due to illness were similar across sites: $15 \%$, Kampala; 17\%, Jinja; 25\%, Mbarara; and 20\%, Mbale.

Of 201,741 clients meeting inclusion criteria, 49\% were female, and $71 \%$ were younger than 30 years of age (Table 1 ). About one half of the clients were single, and about one quarter were seeking premarital testing. The proportion reporting condom use in their last sexual encounter was low but increased from $9 \%$ in 1992 to $19 \%$ in $2000(P<0.0001)$. The distribution of clients by testing site changed over time, with the proportion testing at the Kampala branch decreasing from $70 \%$ in 1992 to
$42 \%$ in 2000 . The proportion seeking premarital testing increased from $24 \%$ to $34 \%$, and those testing as part of a couple increased from $11 \%$ to $30 \%$. These trends were consistent across all sites.

\section{Prevalence of HIV Infection}

Overall, adjusted prevalence of HIV infection declined from $23 \%$ to $13 \%$, with a decrease from $17 \%$ to $9 \%$ among men $(P<0.001)$ and from $31 \%$ to $17 \%$ among women $(P<$ 0.001 ) (Table 2). Declines in unadjusted prevalence were similar, from $17 \%$ to $9 \%$ for men and $31 \%$ to $18 \%$ for women. Annual prevalence of HIV infection was higher among women than among men in every age group. Among men, significant declines were observed in all age groups except those 40 years of age and older. The prevalence appeared stable among men 40 years of age and older. Among women, large declines were observed for women younger than 30 years of age, and a smaller decline was observed for women 30-34 years of age. However, for women 35-39 years old, the prevalence remained stable, and for women 40 years of age and older, the prevalence increased $(P<0.003)$ (Table 2$)$. Although all results here are based on data adjusted for test site, couple testing, and premarital testing, we also analyzed crude prevalence trends and found nearly identical results (data not presented).

The prevalence of HIV infection decreased for all other client subgroups. The prevalence declined from $24 \%$ to $18 \%$ among clients testing as individuals and from $14 \%$ to $7 \%$ among clients testing as part of a couple. The prevalence declined from $18 \%$ to $6 \%$ among clients testing intending to get married and from $25 \%$ to $19 \%$ among others. The prevalence of HIV infection declined at each of the 4 VCT branch sites, and branches with higher initial prevalence of HIV infection showed proportionally larger declines. By ethnic group, which largely reflects geographic region, the prevalence of HIV infection decreased from $25 \%$ to $14 \%$ among Baganda in central Uganda, 16\% to 9\% among the Bagisu in eastern Uganda, 26\% to $14 \%$ among Banyakole in western Uganda, and $16 \%$ to $10 \%$ among the Basoga near Jinja, an industrial town east of Kampala. Declines in the prevalence of HIV infection by district were similar in magnitude to these declines by ethnic group.

\section{Peak Age-Specific Prevalence}

The median age of HIV-positive clients increased from 28 to 34 years for men and from 25 to 29 years for women between 1992 and 2000. Corresponding changes in median age among HIV-negative clients were from 25 to 27 years for men and 22 to 23 years for women. From analysis of the adjusted data, these changes corresponded to a decline in peak age-specific prevalence for both men (31\% to 24\%) and women (44\% to $41 \%)$, whereas the age at which the peak occurred increased for both men (36 to 41 years) and women (31 to 36 years) (Fig. 1). 
TABLE 2. Trends in Adjusted Age-Specific Prevalence of HIV Infection Among Men and Women Attending VCT, 1992-2000

\begin{tabular}{|c|c|c|c|c|c|c|c|c|}
\hline \multirow{2}{*}{$\begin{array}{c}\text { Sex, } \\
\text { Test Year }\end{array}$} & \multicolumn{8}{|c|}{ Age Group } \\
\hline & $15-19$ & $20-24$ & $25-29$ & $30-34$ & 35-39 & $40-44$ & 45 and Older & All Ages \\
\hline \multicolumn{9}{|l|}{ Men } \\
\hline 1992 & $3.7(978)$ & $10.3(3974)$ & $20.3(3166)$ & $27.7(1832)$ & $30.1(661)$ & $27.1(426)$ & $14.7(558)$ & $17.2(11,595)$ \\
\hline 1993 & $2.6(1240)$ & $8.2(5217)$ & $17.2(4702)$ & $25.5(2478)$ & $28.1(1201)$ & $25.7(566)$ & $15.4(735)$ & $15.4(16,139)$ \\
\hline 1994 & $2.2(691)$ & $6.6(3949)$ & $14.6(4058)$ & $22.9(2357)$ & $27.0(1080)$ & $25.8(556)$ & $15.0(667)$ & $14.5(13,360)$ \\
\hline 1995 & $1.6(607)$ & $5.3(3558)$ & $12.4(3728)$ & $20.7(2025)$ & $25.8(1072)$ & $25.5(418)$ & $16.2(604)$ & $13.0(12,014)$ \\
\hline 1996 & $1.3(476)$ & 4.4 (2757) & $10.7(2907)$ & $19.0(1789)$ & $24.1(771)$ & $24.1(384)$ & $15.8(479)$ & $11.9(9570)$ \\
\hline 1997 & $1.0(624)$ & $3.8(2502)$ & $9.9(2729)$ & 17.5 (1397) & $23.0(832)$ & $24.0(340)$ & 14.4 (499) & $10.8(8928)$ \\
\hline 1998 & $0.8(729)$ & $3.0(2860)$ & $8.2(3157)$ & $15.6(1821)$ & $21.4(932)$ & $23.5(503)$ & $14.6(714)$ & $10.2(10,726)$ \\
\hline 1999 & $0.6(718)$ & $2.4(2836)$ & $7.2(2964)$ & $13.9(1782)$ & $21.3(960)$ & $22.9(479)$ & $15.0(668)$ & $9.9(10,434)$ \\
\hline 2000 & $0.5(639)$ & $2.1(2311)$ & $6.1(2516)$ & $13.2(917)$ & $20.0(912)$ & $23.1(482)$ & $15.1(636)$ & $9.1(9201)$ \\
\hline$P$ & $<0.001$ & $<0.001$ & $<0.001$ & $<0.001$ & $<0.001$ & .047 & 0.8 & $<0.001$ \\
\hline All men & $1.7(6702)$ & $5.7(29,964)$ & $12.4(29,927)$ & $20.0(16,398)$ & $24.5(8421)$ & $24.6(4154)$ & $15.1(5560)$ & $12.7(101,967)$ \\
\hline \multicolumn{9}{|l|}{ Women } \\
\hline 1992 & $15.6(2200)$ & $30.4(3227)$ & $41.1(1891)$ & $43.6(1059)$ & $38.6(492)$ & $29.0(249)$ & $14.3(220)$ & $30.6(9396)$ \\
\hline 1993 & $12.5(3142)$ & $27.0(4623)$ & $38.6(2724)$ & $42.7(1622)$ & $38.5(735)$ & $29.9(396)$ & $16.5(281)$ & $28.3(13,644)$ \\
\hline 1994 & $9.5(2971)$ & $22.6(4142)$ & $36.0(2747)$ & $41.9(1680)$ & $39.3(827)$ & $31.5(359)$ & $17.1(339)$ & $26.1(13,128)$ \\
\hline 1995 & $7.4(2896)$ & $19.5(4138)$ & $33.6(2803)$ & $41.0(1601)$ & $39.6(840)$ & $32.6(351)$ & $19.8(318)$ & $24.2(13,033)$ \\
\hline 1996 & $5.7(2579)$ & $16.9(3370)$ & 30.8 (2047) & $39.7(1210)$ & 39.1 (577) & $32.8(318)$ & $20.3(221)$ & $21.3(10,418)$ \\
\hline 1997 & $5.6(2273)$ & $15.7(2869)$ & $28.9(1922)$ & $38.1(917)$ & $38.6(591)$ & $33.5(222)$ & $19.4(270)$ & $20.2(9064)$ \\
\hline 1998 & $4.4(2751)$ & $13.4(3419)$ & $27.4(2393)$ & $37.7(1379)$ & $39.2(875)$ & $35.3(447)$ & $19.3(424)$ & $20.0(11,765)$ \\
\hline 1999 & $3.6(2397)$ & $11.6(3106)$ & $25.2(2021)$ & $36.7(1228)$ & $39.2(767)$ & $36.0(354)$ & $22.8(331)$ & $18.7(10,271)$ \\
\hline 2000 & $3.1(1842)$ & $9.9(2956)$ & $23.1(1865)$ & $35.4(1102)$ & $39.3(585)$ & $36.9(381)$ & $22.4(324)$ & $17.8(9055)$ \\
\hline$P$ & $<0.001$ & $<0.001$ & $<0.001$ & $<0.001$ & 0.8 & 0.003 & 0.003 & $<0.001$ \\
\hline All women & $7.6(23,051)$ & $19.1(31,850)$ & $32.0(20,413)$ & $39.8(11,798)$ & $39.0(6289)$ & $33.2(3077)$ & $19.2(2728)$ & $23.3(99,774)$ \\
\hline \multicolumn{9}{|l|}{ Total } \\
\hline 1992 & $11.9(3178)$ & $19.3(7201)$ & $28.1(5057)$ & $33.5(2891)$ & $33.7(1153)$ & $27.8(675)$ & $14.6(778)$ & $23.2(20,991)$ \\
\hline 1993 & 9.7 (4382) & $17.0(9840)$ & $25.0(7426)$ & $32.3(4100)$ & $32.0(1936)$ & $27.4(962)$ & $15.7(1016)$ & $21.3(29,783)$ \\
\hline 1994 & $8.1(3662)$ & $14.8(8091)$ & $23.2(6805)$ & 30.8 (4037) & $32.3(1907)$ & $28.0(915)$ & $15.7(1006)$ & $20.2(26,488)$ \\
\hline 1995 & $6.3(3503)$ & $13.0(7696)$ & $21.5(6531)$ & $29.7(3626)$ & $31.8(1912)$ & $28.7(769)$ & $17.5(922)$ & $18.8(25,047)$ \\
\hline 1996 & $5.0(3055)$ & $11.3(6127)$ & $19.0(4954)$ & $27.3(2999)$ & 30.5 (1348) & $28.0(702)$ & $17.2(700)$ & $16.7(19,988)$ \\
\hline 1997 & $4.6(2897)$ & $10.1(5371)$ & $17.7(4651)$ & $25.6(2314)$ & $29.5(1423)$ & $27.8(562)$ & $16.2(769)$ & $15.5(17,992)$ \\
\hline 1998 & $3.6(3480)$ & 8.7 (6279) & $16.4(5550)$ & $25.1(3200)$ & $30.0(1807)$ & $29.0(950)$ & $16.3(1138)$ & $15.2(22,491)$ \\
\hline 1999 & $2.8(3115)$ & $7.2(5942)$ & $14.5(4985)$ & $23.2(3010)$ & $29.3(1727)$ & $28.5(833)$ & $17.6(999)$ & $13.9(20,705)$ \\
\hline 2000 & $2.4(2481)$ & $6.4(5267)$ & $13.3(4381)$ & $21.9(2019)$ & $27.5(1497)$ & $29.1(863)$ & $17.6(960)$ & $13.3(18,256)$ \\
\hline$P$ & $<0.001$ & $<0.001$ & $<0.001$ & $<0.001$ & $<0.001$ & 0.4 & 0.08 & $<0.001$ \\
\hline All clients & $6.3(29,753)$ & $12.6(61,814)$ & $20.4(50,340)$ & $28.1(28,196)$ & $30.7(14,710)$ & $28.3(7231)$ & $16.5(8288)$ & $17.9(201,741)$ \\
\hline
\end{tabular}

Data are \% (no.).

\section{ANC and VCT Prevalence Trends}

A comparison of $\mathrm{VCT}$ and $\mathrm{ANC}$ prevalences at 3 major surveillance sites in Uganda showed that both VCT and ANC prevalences declined in a similar fashion overall but had variations by age groups. ${ }^{23}$ Figure 2 illustrates the comparison of crude ANC data and VCT data from Kampala adjusted for test site, couple testing, and premarital testing. For women aged 15-19 years, the prevalence of HIV infection among VCT clients was slightly lower than that among ANC attendees. For women aged 20-24 years, VCT and ANC data appeared almost identical. For older women, the prevalence among AIC
VCT clients was higher than that among ANC attendees. A similar pattern was seen for Jinja and Mbarara, 2 other towns with both ANC sentinel surveillance and VCT data (data not shown). Comparisons before and after adjusting VCT data yielded nearly identical results.

\section{DISCUSSION}

From 1992 to 2000, the prevalence of HIV infection declined from $23 \%$ to $13 \%$ among $>200,000$ first-time clients who sought VCT at Uganda's largest stand-alone VCT provider. Prevalence declines occurred among both men and 


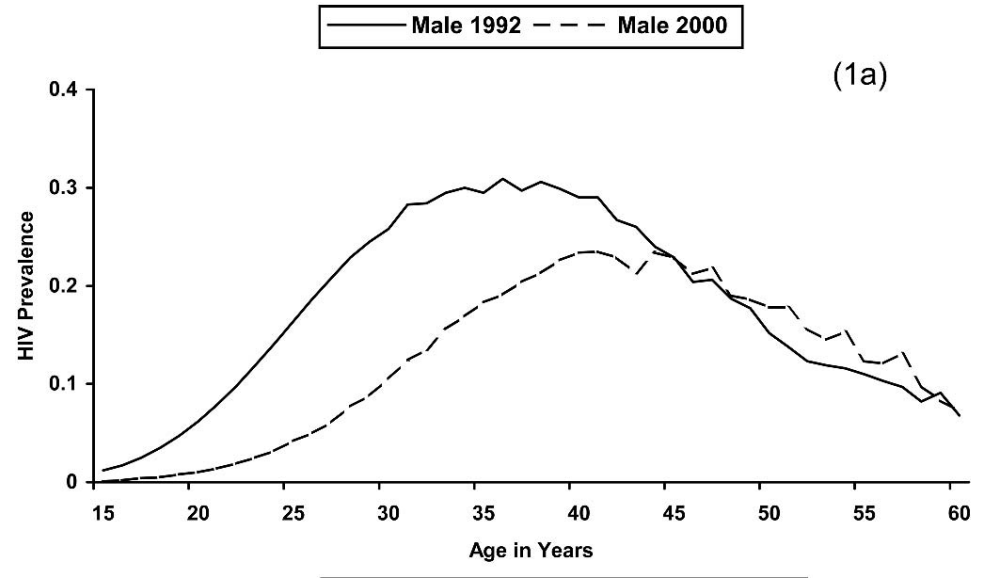

Female $1992 \cdots \cdots \cdot$ - Female 2000

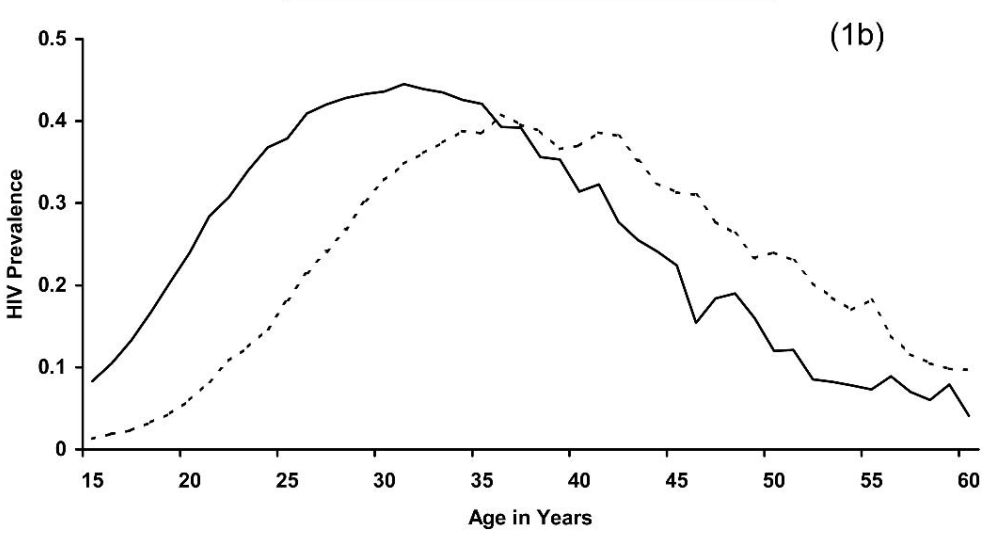

FIGURE 1. Shift in age at peak prevalence of HIV infection among first-time men (a) and women (b) clients receiving VCT at AIC who did not report illness as a reason for taking an HIV test, 1992-2000. women. These findings support surveillance data and population-based cohort data from Uganda showing declining prevalence of HIV infection of a similar magnitude. ${ }^{16,17,23-25} \mathrm{How}-$ ever, although overall prevalence of HIV infection has declined, the prevalence of HIV infection remained high in 2000, particularly in some age groups, and no declines in prevalence were observed in older age groups. In Uganda's mature epidemic, there remains a large burden of prevalent HIV infection in older adults, making them an important target for care and prevention interventions.

\section{Potential Utility of VCT Data as a Supplement to ANC Surveillance}

We found that VCT and ANC prevalence trends were remarkably similar, although the prevalence among VCT attendees was slightly lower for 15- to 19-year-old women and higher for women older than 24 years of age than that among ANC attendees. These findings are consistent with those of studies comparing population-based prevalence data with data for ANC attendees, which showed that due to the effect of HIV on fertility, HIV-infected women are less likely to become pregnant and less likely to carry to term than are women with- out HIV infection. ${ }^{26}$ VCT data may help surveillance systems to overcome a major limitation of ANC data (eg, the underestimation of population-based prevalence among women of childbearing age, particularly in older age groups, and overestimation among younger age groups). In addition, AIC VCT data show marked sex and age differences in the prevalence of HIV infection and trends that ANC data alone could not highlight. These sampling biases and limitations of ANC data may become more pronounced as the epidemic advances.

Although VCT data can be a convenient and useful supplement to standard ANC surveillance data, if data on behavioral and demographic factors are not routinely collected at VCT centers then the utility of VCT data as a monitoring tool may be limited for two reasons. First, in a population that has had an HIV epidemic for $>1$ decade, VCT clientele are more likely to include HIV symptomatic clients. In addition, as VCT becomes more available and accepted, VCT clientele will include increasing proportions of repeat testers, most of whom are HIV negative. If data identifying symptomatic and repeater subgroups are not collected and these groups are not excluded from VCT trend analyses, data interpretation will be difficult. Defining a VCT subpopulation that is comparable over time 
(a)
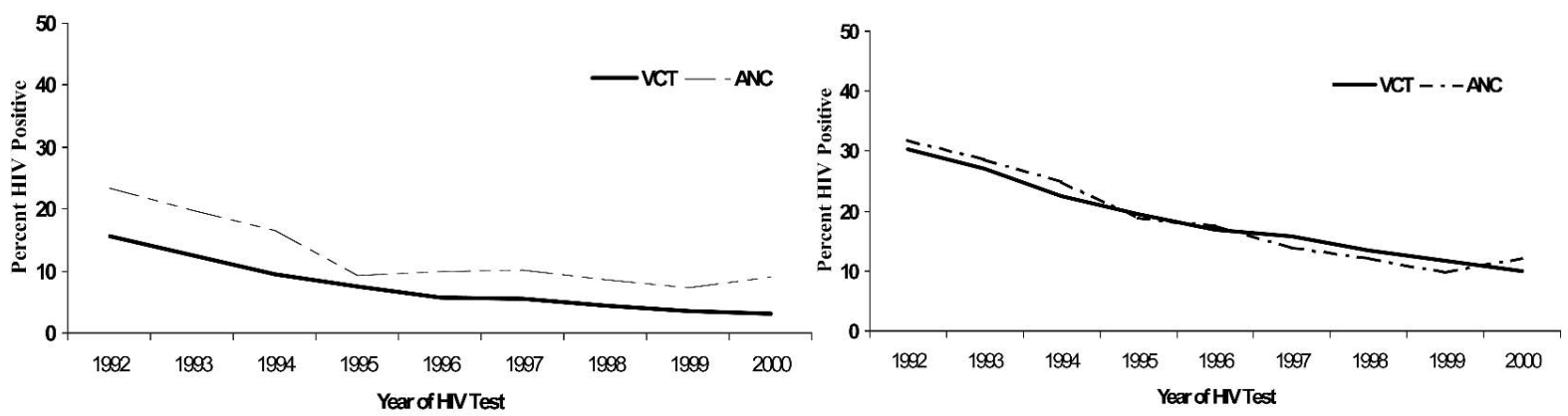

(c) (b)

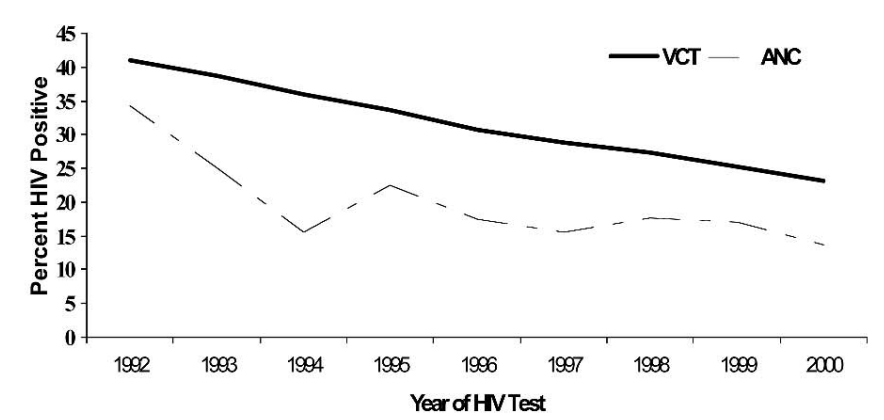

FIGURE 2. Prevalence of HIV infection among ANC attendees at Nsambya Hospital in Kampala, Uganda, compared with adjusted prevalence of HIV infection among women who received VCT at AIC, Kampala branch, in 1992-2000 by age groups: (a) 15-19 years, (b) 20-24 years, and (c) 25-29 years.

and across sites is important. For that reason, we excluded ill VCT clients as well as repeat testers. Exclusion of ill clients would have been more difficult if our VCT data had been drawn from clinic and hospital settings as opposed to standalone VCT sites. Second, our findings underscore the importance of stratifying by age and sex, especially in countries where female prevalence is significantly different from male prevalence and in countries experiencing mature epidemics.

VCT data can be useful for both internal and external monitoring purposes. As VCT services expand, service providers should consider routine data collection tools that will capture key variables such as repeat testing and reason for testing, thus allowing for more accurate interpretation of VCT data. Model data collection tools and data management systems based on VCT experience in several countries in subSaharan Africa are available from the Global AIDS Program at the Centers for Disease Control and Prevention. ${ }^{2}$

\section{Limitations}

Our findings are subject to several limitations. First, VCT clientele may not be representative of the general Ugan- dan population. Persons who seek VCT may be more concerned about their HIV status, reflecting a history of high-risk behavior. Second, our data were drawn from only 4 major towns in Uganda and do not represent the whole country. However, the fact that our data were comparable with ANC data suggests that our major findings of declining prevalence are not likely to have been affected significantly by such differences. Third, characteristics of VCT clientele may have changed over time. If VCT data are to be used as a supplement for surveillance, it will be important to evaluate whether persons who sought testing early in the epidemic are different from persons who tested later in the epidemic and to control for these changes in analyses. However, despite the fact that this analysis was not able to control for all potential biases, our findings appear similar not only to ANC data but also to those of other population-based cohort studies in Uganda where analogous declines have been reported. ${ }^{24,27-29}$

\section{Program Implications}

There are at least 2 important programmatic implications of our findings. First, our results highlight the enormous 
burden that caring for people living with HIV infection poses in the context of a mature epidemic. Peak age-specific prevalences of $41 \%$ among asymptomatic women and $24 \%$ among asymptomatic men in 2000 are very high and would have been much higher had we included ill testers. The bulk of this burden has shifted to considerably older age groups. Integration of preventive therapies such as co-trimoxazole, isoniazid, and antiretroviral therapy into VCT service delivery referral systems could help reduce the burden of care. Although stand-alone VCT has traditionally been seen as an HIV prevention intervention, there will be an increasing need for these VCT service centers to address prevention and treatment of opportunistic infections. Second, although Uganda is the first country in subSaharan Africa to document a decrease in the prevalence of HIV infection, our findings highlight a possible persisting epidemic within the observed declines in prevalence, particularly in older age groups who are not well captured in ANC surveillance systems and who have not been targeted for prevention interventions.

These important findings underscore the usefulness of VCT data not only as a planning tool to target key interventions for HIV prevention and for HIV/AIDS care but also as a tool to help monitor a dynamic and evolving epidemic. In Uganda, where large-scale stand-alone VCT has occurred for $>1$ decade, VCT data appear to be an excellent supplement to standard ANC surveillance data. Triangulation of ANC data with VCT, PMTCT, and other programmatic data could allow for enhanced surveillance as HIV prevention and care interventions expand. VCT data should be considered in other settings as a surveillance tool, and international guidelines for VCT data collection, analysis, and interpretation should be developed.

\section{ACKNOWLEDGMENTS}

The authors thank the AIC, Uganda, for their support for this work. They also thank Dr. Robert Downing who supervised AIC HIV testing and thoughtfully reviewed this manuscript and Dr. Stefan Wiktor and Dr. Meade Morgan for their careful reviews.

\section{REFERENCES}

1. Grulich AE, Kaldor JM. Evidence of success in HIV prevention in Africa. Lancet. 2002;360:3-4.

2. CDC Global AIDS Program. 2002. Available at: http://www.cdc.gov/ achet/ppif.

3. Oberzaucher N, Baggaley R. 2002. HIV voluntary counseling and testing: a gateway to prevention and care, UNAIDS Case Study, Geneva.

4. de Cock KM, Mbori-Ngacha D, Marum E. Shadow on the continent: public health and HIV/AIDS in Africa in the 21st century. Lancet. 2002;360: 67-72.

5. Weidle P, Mastro TD, Grant AD, et al. HIV/AIDS treatment and HIV vaccines for Africa. Lancet. 2002;359:2261-2267.
6. Marseille E, Kahn JG, Mmiro F, et al. Cost effectiveness of single-dose nevirapine regimen for mothers and babies to decrease vertical HIV-1 transmission in sub-Saharan Africa. Lancet. 1999;354:803-809.

7. Guay LA, Musoke P, Fleming T, et al. Intrapartum and neonatal singledose nevirapine compared with zidovudine for prevention of mother-tochild transmission of HIV-1 in Kampala, Uganda: HIVNET 012 randomised trial. Lancet. 1999;354:795-802.

8. Sweat M, Gregorich S, Sangiwa G, et al. Cost-effectiveness of voluntary HIV-1 counseling and testing in reducing sexual transmission of HIV-1 in Kenya and Tanzania. Lancet. 2000;356:113-121.

9. Glynn JR, Buve A, Carael M, et al. Factors influencing the difference in HIV prevalence between antenatal clinic and general population in subSaharan Africa. AIDS. 2001;15:1717-1725.

10. UNAIDS. 2002. Report on the global HIV/AIDS epidemic, Geneva.

11. Population Reference Bureau. World population data sheet of the Population Reference Bureau. 2003. Available at: http://www.prb.org.

12. Ministry of Health. Policy for Reduction of the Mother-to-Child HIV Transmission 2001, Kampala, Uganda.

13. Johnstone F, Goldberg D, Tappin D, et al. The incidence and prevalence of HIV infection among childbearing women living in Edinburgh city, 1982-1995. AIDS. 1998;12:911-917.

14. Zaba B, Boerma T, White R. Monitoring the AIDS epidemic using HIV prevalence data among young women attending antenatal clinics: prospects and problems. AIDS. 2000;14:1633-1645.

15. Kigadye RM, Klokke A, Nicoll A, et al. Sentinel surveillance for HIV-1 among pregnant women in a developing country: 3 years' experience and comparison with a population serosurvey. AIDS. 1993;7:849-855.

16. Kilian AHD, Gregson S, Ndyanabangi B, et al. Reduction in risk behavior provides the most consistent explanation for declining HIV-1 prevalence in Uganda. AIDS. 1999;13:391-398.

17. Stoneburner RL, Low-Beer D, Tembo GS, et al. Human immunodeficiency virus infection in East Africa deduced from surveillance data. Am J Epidemiol. 1996;144:682-695.

18. Bunnell RE, Yanpaisarn S, Kilmarx PH, et al. HIV-1 seroprevalence among childbearing women in northern Thailand: monitoring a rapidly evolving epidemic. AIDS. 1999;13:509-515.

19. Painter TM. Voluntary counseling and testing for couples: a highleverage intervention for HIV/AIDS prevention in sub-Saharan Africa. Soc Sci Med. 2001;53:1397-1411.

20. McKenna SL, Muyinda GK, Roth D, et al. Rapid HIV testing and counseling for voluntary testing centers in Africa. AIDS. 1997;11:S103-S110.

21. Knowledge is Power: Voluntary HIV counseling and testing in Uganda. UNAIDS, 1999, UNAIDS Case Study, Geneva.

22. Downing RG, Otten RA, Marum E, et al. Optimizing the delivery of HIV counseling and testing services: the Uganda experience using rapid HIV antibody test algorithms. J Acquir Immune Defic Syndr Hum Retrovirol. 1998;18:384-388.

23. Ministry of Health. HIV/AIDS Surveillance Report. 2001, Kampala, Uganda.

24. Kamali A, Carpenter LM, Whitworth JAG, et al. Seven-year trends in HIV-1 infection rates, and changes in sexual behavior, among adults in rural Uganda. AIDS. 2000;14:427-433.

25. Sewankambo NK, Wawer MJ, Gray RH, et al. Demographic impact of HIV infection in rural Rakai district, Uganda: results of a populationbased cohort study. AIDS. 1994;8:1707-1713.

26. Gray RH, Wawer MJ, Serwadda D, et al. Population-based study of fertility in women with HIV-1 infection in Uganda. Lancet. 1998;351:98103.

27. Fabiani M, Accorsi S, Lukwiya M, et al. Trend in HIV-1 prevalence in an antenatal clinic in north Uganda and adjusted rates for the general female population. AIDS. 2001;15:97-103.

28. Mulder D, Nunn A, Kamali A, et al. Decreasing HIV-1 seroprevalence in young adults in a rural Ugandan cohort. BMJ. 1995;311:833-836.

29. Wawer MJ, Serwadda D, Gray RH, et al. Trends in HIV-1 prevalence may not reflect trends in incidence in mature epidemics: data from the Rakai population-based cohort, Uganda. AIDS. 1997;11:1023-1030. 